\title{
Surgical challenge in portocaval shunt: A case of budd chairi syndrome
}

\author{
Rohith MUDADLA* \\ Institute of Surgical Gastroenterology and Liver Transplantation, Stanley Medical College, \\ The Tamilnadu DR. MGR University of Medical Sciences, Chennai, India
}

Introduction: The Budd chairi syndrome caused by occlusion of the major hepatic veins often of unknown etiology is typically characterized by massive ascites, hepatomegaly, abdominal pain due to congestion of the liver. The long-term outcome of these patients is fatal without an improper management. In a significant proportion of patients, surgical decompressive procedure (shunt operations) are effective in decongesting the liver and improving the survival before on set of end stage liver disease or cirrhosis. SSPCS side to side portocaval shunt proved to be most widely applied and durable.

Methods: A 26-year-old male presented with chief complaints of painless progressive abdominal distension and loss of appetite since 4 months. He was evaluated as a case of chronic budd chairi syndrome with out signs of hepatic decompensation and was planned for side to side portocaval shunt. Due to extensive caudate lobe hypertrophy IVC-PV could not be approximated, in such challenging situation intraoperatively he was planned for partial/complete caudate lobe excision, during which there was torrential bleed from retroperitoneal collaterals, and procedure to excise caudate lobe was abandoned.

Results: Portocaval interposition shunting was performed using PTFE graft. On follow up for 18 months, flow across the graft was patent, there was no signs of jaundice or ascites.

Conclusions: Shunt surgeries still offer better outcomes and long term survival in patients with preserved liver function where organ donor for liver transplantation is limited. Though SSPCS has best outcome for BCS in studies, interposition grafts can be used in unpredictable and difficult situations with graft patency rate of almost $52 \%$ in 5 years. 